\title{
The $N$-intertwined SIS epidemic network model
}

\author{
Piet Van Mieghem
}

Received: 21 September 2011 / Accepted: 27 September 2011 / Published online: 13 October 2011 (C) The Author(s) 2011. This article is published with open access at Springerlink.com

\begin{abstract}
Serious epidemics, both in cyber space as well as in our real world, are expected to occur with high probability, which justifies investigations in virus spread models in (contact) networks. The $N$-intertwined virus spread model of the SIS-type is introduced as a promising and analytically tractable model of which the steady-state behavior is fairly completely determined. Compared to the exact SIS Markov model, the $N$-intertwined model makes only one approximation of a mean-field kind that results in upper bounding the exact model for finite network size $N$ and improves in accuracy with $N$. We review many properties theoretically, thereby showing, besides the flexibility to extend the model into an entire heterogeneous setting, that much insight can be gained that is hidden in the exact Markov model.
\end{abstract}

Keywords Epidemics $\cdot$ Networks $\cdot$ Robustness $\cdot$ Mean-field approximation

Mathematics Subject Classification (2000) $\quad 05 \mathrm{C} 50 \cdot 05 \mathrm{C} 82 \cdot 37 \mathrm{H} 20 \cdot 46 \mathrm{~N} 10$. $46 \mathrm{~N} 30 \cdot 60 \mathrm{~J} 28$

\section{Introduction}

We investigate the influence of the network topology on the spread of viruses, whose dynamics is modeled by a susceptible-infected-susceptible (SIS) type of process. The SIS disease model $[1,3,10]$ can be regarded as one of the simplest virus infection models, in which persons or nodes in a network are either in two states: "healthy, but susceptible to infection" or "infected by the disease or virus and, thus, infectious to neighbors". In this article, the word "virus" is understood in the most general wording possible as an "item" transferred from the surroundings towards a node in the network.

P. Van Mieghem $(\varangle)$

Faculty of Electrical Engineering, Mathematics and Computer Science,

Delft University of Technology, P.O. Box 5031, 2600 GA, Delft, The Netherlands

e-mail: P.F.A.VanMieghem @ tudelft.nl 
At a node, the "item" can be destroyed, but can also be propagated to neighbors of that node. For example, digital viruses (of all kind, and generally called malware) are living in cyberspace and use mainly the Internet as the transport media, while biological viruses contaminate other living beings and use "contacts" among their victims as their propagation network. A digital "virus" here can also mean a rumor, news or any kind of information that spreads over a data communications or social network. Recently, Hill et al. [17] have considered happiness of persons as a form of social infection and have modeled the spread of emotions over the social contact network as a SIS-type of epidemic.

Our SIS, continuous-time model for the spreading of a virus in a network, called the $N$-intertwined virus spread model [36], is reviewed in Sect. 2. It was earlier considered by Ganesh et al. [13] and by Wang et al. [40] in discrete-time, whose paper was later improved in [7] after which their discrete-time model also appeared in the physics community [15]. An infected node can infect its neighbors with an infection rate $\beta$ (per link), but it is cured with curing rate $\delta$ (per node). However, once cured and healthy, the node is again prone to the virus. Both infection and curing processes are independent. There exists a wealth of variants or refinements of the SIS model (see e.g. [2,10,14, 21,41]): there can be an incubation period, an infection rate that depends on the number of neighbors or that has a constant component (as in [17]), a curing process that takes a certain amount of time, and many other sophistications that we do not consider here. Whereas most of the recent contributions to epidemics in networks were made by physicists, Durrett [12] doubts about the mathematical rigor of many of their analyses.

A remarkable property of the SIS model is the appearance of a phase-transition $[4,6]$ when the effective infection rate $\tau=\frac{\beta}{\delta}$ approaches the epidemic threshold $\tau_{c}=\frac{1}{\lambda_{1}}$, where $\lambda_{1}$ is the largest eigenvalue of the adjacency matrix $A$, also called the spectral radius. Below the epidemic threshold, $\tau<\tau_{c}$, the network is virus-free in the steady-state, while for $\tau>\tau_{c}$, there is always a fraction of nodes that remains infected. For the companion infection model, SIR, where R stands for recovered or removed, Kermack and McKendrick [19] have shown that no epidemic can occur if the population density is below a critical threshold. Recently, Youssef and Scoglio [43] have extended the $N$-intertwined SIS model to SIR epidemics and they have shown that the SIR-epidemic threshold also equals $\tau_{c \text {; SIR }}=\frac{1}{\lambda_{1}}$. We point here to another dynamic process on a network, that bears resemblance to virus spread. The synchronization of coupled oscillators in a network features a surprisingly similar phase transition: the onset of oscillator coupling occurs [28] at a critical coupling strength $g_{c}=\frac{g_{0}}{\lambda_{1}}$ and the behavior of the phase transition around $g_{c}$ is mathematically similar [31]. Synchronization [29] plays a role in sensor networks, human body (heart beat, brain, epilepsy), light emission of fire-flies, etc.

The major goal of this article is to provide a consistent, theoretical overview of the steady-state in the $N$-intertwined virus spread model, that we consider as almost completely solved since the recent solution [31] of the behavior around the epidemic threshold. Section 2 explains the $N$-intertwined SIS virus spread model and the meanfield approximation. From Sect. 3 on, we confine to the steady-state of the $N$-intertwined SIS model and present general equations for the fraction $y_{\infty}$ of steady-state infected nodes, from which the continued fraction expansion is derived. In addition, 
two series for $y_{\infty}$ are given, whose coefficients obey recursion relations, specified in Lemmas 1 and 3. Section 3.2 specifies the condition for the epidemic threshold $\tau_{c}=\frac{1}{\lambda_{1}}$ and the behavior of $y_{\infty}(\tau)$ around $\tau_{c}$, with references to many approaches in networks to enlarge the epidemic threshold. After arguing that the transform $s=\frac{1}{\tau}$ is more natural, bounds on $y_{\infty}(s)$ around $s=\frac{2}{E[D]}$ are presented in Sect. 4 inspired by simulations in [42] indicating that $y_{\infty}(s)$ is close to $\frac{1}{2}$ for $s=\frac{2}{E[D]}$. Section 5 discusses the viral conductance $\psi$ of a virus spreading process in a graph, that was first proposed in [20]. We end our review by extending the $N$-intertwined model to a heterogeneous setting in Sect. 6: the governing equation and the continued fraction are readily found, while the convexity Theorem 5 has interesting applications in network protection strategies $[16,26]$. Section 7 concludes with an outlook on open problems.

\section{The $N$-intertwined SIS model}

A network is represented by an undirected graph $G(N, L)$ with $N$ nodes and $L$ links. The network topology is described by a symmetric adjacency matrix $A$, in which the element $a_{i j}=a_{j i}=1$ if there is a link between nodes $i$ and $j$, otherwise $a_{i j}=0$. In the sequel, we confine ourselves and make the following simplifying assumptions. The state of a node $i$ is specified by a Bernoulli random variable $X_{i} \in\{0,1\}: X_{i}=0$ for a healthy node and $X_{i}=1$ for an infected node. A node $i$ at time $t$ can be in one of the two states: infected, with probability $v_{i}(t)=\operatorname{Pr}\left[X_{i}(t)=1\right]$ or healthy, with probability $1-v_{i}(t)$. We assume that the curing process per node $i$ is a Poisson process with rate $\delta$, and that the infection rate per link is a Poisson process with rate $\beta$. All involved Poisson processes are independent. The effective infection rate is defined as $\tau=\frac{\beta}{\delta}$. We assume that the initial infection state $v_{i}(0)$ in each node $i$ is known. This is the general description of the simplest type of a SIS virus spread model in a network and the challenge is to determine the virus infection probability $v_{i}(t)$ for each node $i$ in the graph $G$ at time $t$.

This SIS model can be expressed exactly in terms of a continuous-time Markov model with $2^{N}$ states as shown in [36]. Unfortunately, the exponentially increasing state space with $N$ prevents the determination of the set of $\left\{v_{i}(t)\right\}_{1 \leq i \leq N}$ in realistic networks, which has triggered a spur of research to find good approximate solutions. For an overview of SIS heuristics and numerous extensions, we refer to [4,21,42].

In contrast to all published SIS-type of models, the $N$-intertwined model, proposed and investigated in depth in [36], only makes one (mean-field) approximation in the exact SIS model and is applicable to all graphs.

\subsection{The mean-field approximation}

By separately observing each node, every node $i$ at time $t$ in the network has two states: infected with probability $v_{i}(t)=\operatorname{Pr}\left[X_{i}(t)=1\right]$ and healthy with probability $\operatorname{Pr}\left[X_{i}(t)=0\right]=1-v_{i}(t)$. If we apply Markov theory straight away, the infinitesimal generator $Q_{i}(t)$ of this two-state continuous Markov chain is,

$$
Q_{i}(t)=\left[\begin{array}{cc}
-q_{1 ; i} & q_{1 ; i} \\
q_{2 ; i} & -q_{2 ; i}
\end{array}\right]
$$




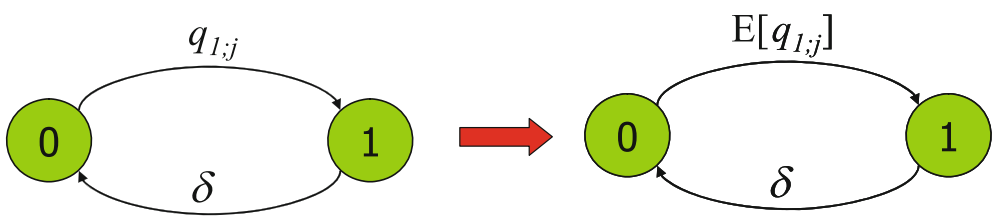

Fig. 1 The mean-field approximation (arrow) transforms the random variable $q_{1 ; j}$ into the average $E\left[q_{1 ; j}\right]$. Instead of being infected at time $t$ by the precise number of infected neighbors, the node $i$ is now infected by the average number of infected neighbors

with $q_{2 ; i}=\delta$ is the curing rate and

$$
q_{1 ; i}=\beta \sum_{j=1}^{N} a_{i j} 1_{\left\{X_{j}(t)=1\right\}}
$$

where the indicator function $1_{x}=1$ if the event $x$ is true else it is zero. The rate $q_{1 ; i}$ equals the sum over all infection rates of infected neighbors of node $i$ and this rate $q_{1 ; i}$ couples or "intertwines" node $i$ to the rest of the network through the appearance of the events $\left\{X_{j}(t)=1\right\}$. As mentioned in [36], the total infection rate $q_{1 ; i}$ is a random variable, whereas ordinary Markov theory requires that $q_{1 ; j}$ is a real number. The random nature of $q_{1 ; i}$ is removed by an additional conditioning to all possible combinations of rates, which is equivalent to conditioning to all possible combinations of the states $X_{j}(t)=1$ (and their complements $X_{j}(t)=0$ ) of the neighbors of node $i$. Hence, the number of basic states in the Markov process dramatically increases from two states per node to all possible combinations of states for $N$ nodes. Eventually, after conditioning each node in such a way, we end up with the exact $2^{N}$-state Markov chain, specified in [36].

Instead of conditioning, the mean-field approximation consists of replacing $q_{1 ; i}$ by its average $E\left[q_{1 ; i}\right]$, which is a real number and allows immediate application of continuous-time Markov theory [30]. Figure 1 illustrates the mean-field approximation. Using $E\left[1_{x}\right]=\operatorname{Pr}[x]$, we replace $q_{1 ; i}$ by

$$
E\left[q_{1 ; i}\right]=\beta \sum_{j=1}^{N} a_{i j} \operatorname{Pr}\left[X_{j}(t)=1\right]=\beta \sum_{j=1}^{N} a_{i j} v_{j}(t)
$$

which results in an effective infinitesimal generator,

$$
\overline{Q_{i}(t)}=\left[\begin{array}{cc}
-E\left[q_{1 ; i}\right] & E\left[q_{1 ; i}\right] \\
\delta & -\delta
\end{array}\right]
$$

Due to the dependence of $E\left[q_{1 ; i}\right]$ on $v_{j}(t)$, the Markov differential equation [30, (10.11) on p. 182] for state $X_{i}(t)=1$ turns out to be non-linear, 


$$
\frac{d v_{i}(t)}{d t}=\beta\left(1-v_{i}(t)\right) \sum_{j=1}^{N} a_{i j} v_{j}(t)-\delta v_{i}(t)
$$

The governing differential equation (1) in the $N$-intertwined model for a node $i$ has the following physical interpretation: the time-derivative of the infection probability of a node $i$ consists of two competing processes: (1) while healthy with probability $\left(1-v_{i}(t)\right)$, all infected neighbors, an event with probability $\sum_{j=1}^{N} a_{i j} v_{j}(t)$, try to infect the node $i$ with rate $\beta$ and (2) while infected with probability $v_{i}(t)$, the node $i$ is cured at rate $\delta$. This rather intuitive explanation has been directly used in former models such as the Kephart and White model [18] to derive the differential equation, thereby implicitly making a mean-field approximation.

Defining the vector $V(t)=\left[v_{1}(t) v_{2}(t) \cdots v_{N}(t)\right]^{T}$, the matrix representation based on (1) becomes

$$
\frac{d V(t)}{d t}=(\beta A-\delta I) V(t)-\beta \operatorname{diag}\left(v_{i}(t)\right) A V(t)
$$

where diag $\left(v_{i}(t)\right)$ is the diagonal matrix with elements $v_{1}(t), v_{2}(t), \ldots, v_{N}(t)$. We define the (average) fraction of infected nodes in the network at time $t$ as

$$
y(t)=\frac{1}{N} E\left[\sum_{j=1}^{N} 1_{\left\{X_{j}(t)=1\right\}}\right]=\frac{1}{N} \sum_{j=1}^{N} v_{j}(t)
$$

In $[25,36]$, we show that the mean-field approximation implies that (a) the $N$-intertwined model upperbounds the exact probability $v_{i}(t)$ of infection, (b) the deviations between the $N$-intertwined and the exact model are largest for intermediate values of $\tau$ around $\tau_{c}$ and (c) the random variables $X_{j}$ and $X_{i}$ are implicitly assumed to be independent. Since the latter basic assumption is increasingly good for large $N$, we expect that the deductions from the $N$-intertwined model are asymptotically (for $N \rightarrow \infty$ ) almost exact for real-world networks.

Figure 2 shows 500 sample paths of the exact SIS process, together with the steadystate fraction $y_{\infty}(\tau)$ of infected nodes. Although the steady-state fraction is constant, the SIS infection process continues for ever, which means that an arbitrary node $i$ moves between the healthy and infected state for $1-v_{i \infty}$ and $v_{i \infty}$ percent of the time, respectively.

\section{The steady-state fraction $y_{\infty}$ of infected nodes}

In this section, we focus on the steady-state of the $N$-intertwined model, where $v_{i \infty}=\lim _{t \rightarrow \infty} v_{i}(t)$ and $\lim _{t \rightarrow \infty} \frac{d v_{i}(t)}{d t}=0$. The corresponding steady-state vector is denoted by $V_{\infty}$. In the exact SIS model, the steady-state is the healthy state, which is the only absorbing state in the Markov process. However, in networks of realistic size $N$, this steady-state is only reached after an unrealistically long time [13]. The 


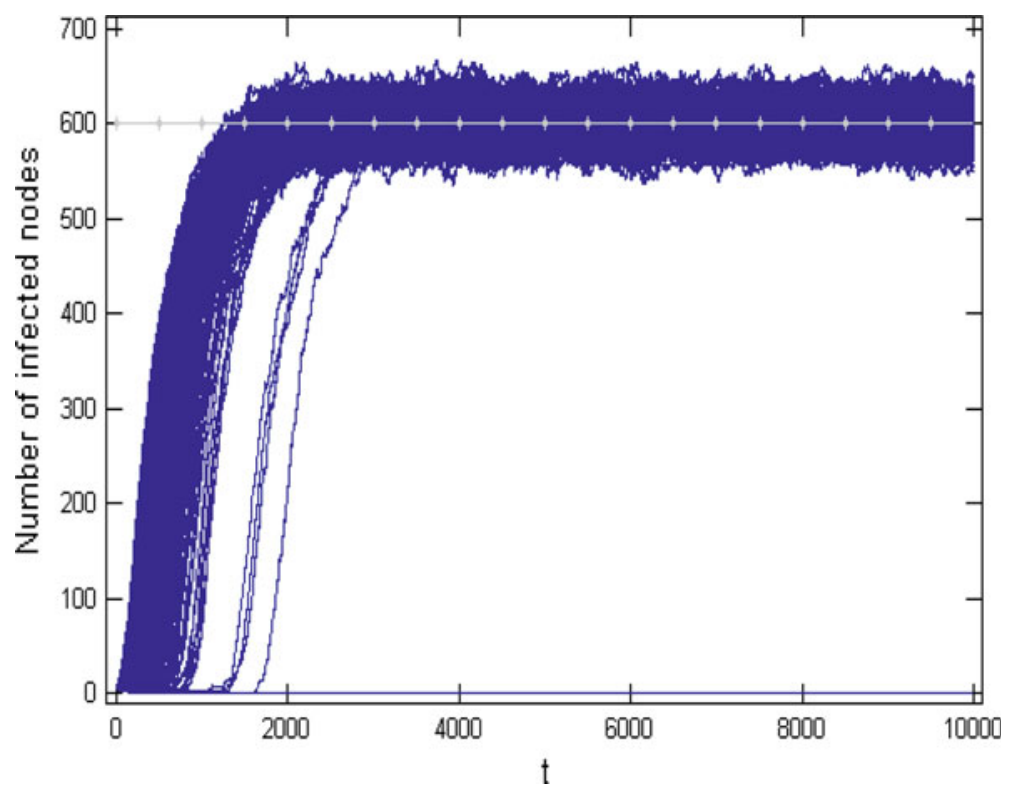

Fig. 2 A time-dependent simulation of the exact SIS number of infected nodes $N y(t ; \tau)$ in the complete bipartite graph $K_{10,990}$ for $\tau=0.15$. The steady-state fraction (8) of infected nodes in the $N$-intertwined model gives $y_{\infty}(0.15)=0.6$, which is the mean value of the 500 realizations of the spreading process

steady-state in the $N$-intertwined virus spread model thus refers to the "metastable state", which is reached exponentially fast and which reflects real epidemics more closely. In fact, the mean-field approximation, which transforms the linear set of $2^{N}$ differential equations of the exact Markov chain into a set of $N$ non-linear differential equations in (2), has induced the existence of the "metastable state" as well as the phase transition and the corresponding epidemic threshold, discussed in Sect. 3.2.

From (1), we obtain with $\frac{d v_{i}(t)}{d t}=0$ and $v_{i \infty}=\lim _{t \rightarrow \infty} v_{i}(t)$,

$$
v_{i \infty}=\frac{\beta \sum_{j=1}^{N} a_{i j} v_{j \infty}}{\beta \sum_{j=1}^{N} a_{i j} v_{j \infty}+\delta}=1-\frac{1}{1+\tau \sum_{j=1}^{N} a_{i j} v_{j \infty}}
$$

Beside the trivial solution $v_{i \infty}=0$, (4) illustrates that there is another positive solution reflecting the metastable state in which we are interested here. For regular graphs, where each node has degree $d$, symmetry in the steady-state implies that $v_{i \infty}=v_{\infty}$ for all nodes $i$ and it follows from (4) with the definition of the degree $d_{i}=\sum_{j=1}^{N} a_{i j}$ that

$$
v_{\infty ; \text { regular }}=y_{\infty ; \text { regular }}(\tau)=1-\frac{1}{\tau d}
$$

where $y_{\infty}=\frac{1}{N} \sum_{i=1}^{N} v_{i \infty}$ is the fraction of infected nodes in the steady-state as deduced from the definition (3). Besides the regular graph, [36] gives an exact 
solution of the steady-state infection behavior in the complete bipartite graph $K_{m, n}$, where there are two partitions $\mathcal{N}_{m}$ with $m$ nodes and $\mathcal{N}_{n}$ with $n$ nodes [32] so that $N=m+n$ and $L=m n$,

$$
v_{i \infty}=\frac{m n-\frac{1}{\tau^{2}}}{\left(\frac{1}{\tau}+m\right) n} \quad i \in \mathcal{N}_{n}
$$

and

$$
v_{j \infty}=\frac{m n-\frac{1}{\tau^{2}}}{\left(\frac{1}{\tau}+n\right) m} \quad j \in \mathcal{N}_{m}
$$

Thus, since $y_{\infty}=\frac{n v_{i \infty}+m v_{j \infty}}{n+m}$, we obtain

$$
y_{\infty}(\tau)=\frac{\left(m n-\tau^{-2}\right)}{N}\left\{\frac{1}{\tau^{-1}+m}+\frac{1}{\tau^{-1}+n}\right\}
$$

The complete bipartite graph, of which the star $K_{1, n}$ is a special case, often appears as our benchmark model (see e.g. [22,23,33]).

The nodal infection steady-state equation (4) can be solved, as proved in [36] and alternatively in [33]:

Theorem 1 For any effective spreading rate $\tau=\frac{\beta}{\delta} \geq 0$, the non-zero steady-state infection probability of any node $i$ in the $N$-intertwined model can be expressed as a continued fraction

$$
v_{i \infty}=1-\frac{1}{1+\tau d_{i}-\tau \sum_{j=1}^{N} \frac{a_{i j}}{1+\tau d_{j}-\tau \sum_{k=1}^{N} \frac{a_{j k}}{1+\tau d_{k}-\tau \sum_{q=1}^{N} \frac{a_{k q}}{1+\tau d_{q}-} \cdot}}}
$$

where $d_{i}=\sum_{j=1}^{N} a_{i j}$ is the degree of node $i$. Consequently, the exact steady-state infection probability of any node $i$ is bounded by

$$
0 \leq v_{i \infty} \leq 1-\frac{1}{1+\tau d_{i}}
$$

The interesting feature of the continued fraction (9) is that each convergent is an upper bound for $v_{i \infty}$. Another, equally useful, representation for $v_{i \infty}$ is the Laurent series, proved in [33]:

Lemma 1 The Laurent series of the steady-state infection probability

$$
v_{i \infty}(\tau)=1+\sum_{m=1}^{\infty} \eta_{m}(i) \tau^{-m}
$$


possesses the coefficients

$$
\eta_{1}(i)=-\frac{1}{d_{i}}
$$

and

$$
\eta_{2}(i)=\frac{1}{d_{i}}\left(\frac{1}{d_{i}}+\sum_{j=1}^{N} \frac{a_{i j}}{d_{j}}\right)
$$

and for $m \geq 2$, the coefficients obey the recursion

$$
\eta_{m+1}(i)=-\frac{1}{d_{i}}\left\{\eta_{m}(i)\left\{1-\sum_{j=1}^{N} \frac{a_{i j}}{d_{j}}\right\}+\sum_{k=2}^{m} \eta_{m+1-k}(i) \sum_{j=1}^{N} a_{i j} \eta_{k}(j)\right\}
$$

Consequently, the Laurent series for the steady-state fraction of infected nodes equals

$$
y_{\infty}(\tau)=1+\frac{1}{N} \sum_{m=1}^{\infty}\left\{\sum_{i=1}^{N} \eta_{m}(i)\right\} \tau^{-m}
$$

\subsection{General relations for $y_{\infty}$}

Summing (1) over all $i$ is equivalent to right multiplication of $V(t)$ by the all one vector $u^{T}$ because $\sum_{i=1}^{N} v_{i}(t)=u^{T} V(t)$. Then, we find from (2) that

$$
\begin{aligned}
\frac{d u^{T} V(t)}{d t} & =u^{T}\left(\operatorname{diag}\left(1-v_{i}(t)\right) \beta A-\delta I\right) V(t) \\
& =\beta(u-V(t))^{T} A V(t)-\delta u^{T} V(t)
\end{aligned}
$$

Hence, we obtain a relation for $y_{\infty} \in[0,1]$ in terms of the vector $V_{\infty}$ :

$$
N y_{\infty}=u^{T} V_{\infty}=\tau\left(u-V_{\infty}\right)^{T} A V_{\infty}
$$

Since $u^{T} A=D^{T}$ because $A=A^{T}$, we can write (16) as

$$
y_{\infty}(\tau)=\frac{\tau}{N}\left(D^{T} V_{\infty}-V_{\infty}^{T} A V_{\infty}\right)
$$

We write the degree vector $D$ as $D=\Delta u$, where $\Delta=\operatorname{diag}\left(d_{1}, d_{2}, \ldots, d_{N}\right)$, so that

$$
\begin{aligned}
N y_{\infty} & =\tau\left(u^{T} \Delta V_{\infty}+V_{\infty}^{T} \Delta V_{\infty}-V_{\infty}^{T} \Delta V_{\infty}-V_{\infty}^{T} A V_{\infty}\right) \\
& =\tau\left(\left(u-V_{\infty}\right)^{T} \Delta V_{\infty}+V_{\infty}^{T}(\Delta-A) V_{\infty}\right)
\end{aligned}
$$


Introducing the Laplacian $Q=\Delta-A$ of the graph $G$, the steady-state fraction of infected nodes $y_{\infty}$ is expressed as a quadratic form in terms of the Laplacian,

$$
y_{\infty}=\frac{\tau}{N}\left(\left(u-V_{\infty}\right)^{T} \Delta V_{\infty}+V_{\infty}^{T} Q V_{\infty}\right)
$$

After left-multiplication of the steady state version of (2) by the vector

$$
V_{\infty}^{T} \operatorname{diag}\left(v_{i \infty}^{k-1}\right)=\left[v_{1 \infty}^{k} v_{2 \infty}^{k} \cdots v_{N \infty}^{k}\right]^{T}
$$

which we denote by $\left(V_{\infty}^{k}\right)^{T}$, we obtain the scalar

$$
\left(V_{\infty}^{k}\right)^{T} V_{\infty}=\sum_{j=1}^{N} v_{j \infty}^{k+1}=\tau\left(\left(V_{\infty}^{k}\right)^{T} A V_{\infty}-\left(V_{\infty}^{k+1}\right)^{T} A V_{\infty}\right)
$$

For $k=0$ in (19), and introducing the all one vector $u=\lim _{k \rightarrow 0} V_{\infty}^{k}$, we obtain (16) again. For $k=1$ in (19), the norm $\left\|V_{\infty}\right\|_{2}^{2}=V_{\infty}^{T} V_{\infty}=\sum_{j=1}^{N} v_{j \infty}^{2}$ obeys

$$
V_{\infty}^{T} V_{\infty}=\tau\left(V_{\infty}^{T} A V_{\infty}-V_{\infty}^{T} \operatorname{diag}\left(v_{i \infty}\right) A V_{\infty}\right)
$$

When summing (19) over all $k$ from $m \geq 0$ to infinity and taking $\left|v_{j \infty}\right|<1$ into account, the telescoping nature of the right-hand side leads to

$$
\sum_{k=m}^{\infty}\left(V_{\infty}^{k}\right)^{T} V_{\infty}=\sum_{j=1}^{N} \frac{v_{j \infty}^{m+1}}{1-v_{j \infty}}=\tau\left(V_{\infty}^{m}\right)^{T} A V_{\infty}
$$

When $m=0$, we have that $V_{\infty}^{m}=u$ and we obtain, with the degree vector $u^{T} A=D^{T}$,

$$
\frac{1}{\tau} \sum_{j=1}^{N} \frac{v_{j \infty}}{1-v_{j \infty}}=D^{T} V_{\infty}=\sum_{j=1}^{N} d_{j} v_{j \infty}
$$

As shown earlier in [36], the characteristic structure (21) of the $N$-intertwined model follows more elegantly from the governing equation (2) in the steady-state

$$
V_{\infty}=\tau \operatorname{diag}\left(1-v_{i \infty}\right) A V_{\infty}
$$

for finite $\tau$ such that $v_{i \infty}<1$. Indeed, after left-multiplying both sides by $\left(\operatorname{diag}\left(1-v_{i \infty}\right)\right)^{-1}=\operatorname{diag}\left(\frac{1}{1-v_{i \infty}}\right)$, we have

$$
\frac{1}{\tau} \operatorname{diag}\left(\frac{1}{1-v_{i \infty}}\right) V_{\infty}=A V_{\infty}
$$


or

$$
\frac{1}{\tau} \frac{V_{\infty}}{1-V_{\infty}}=A V_{\infty}
$$

where the vector $\left(\frac{V_{\infty}}{1-V_{\infty}}\right)^{T}=\left[\frac{v_{1 \infty}}{1-v_{1 \infty}} \frac{v_{2 \infty}}{1-v_{2 \infty}} \cdots \frac{v_{N \infty}}{1-v_{N \infty}}\right]^{T}$. By left-multiplication of (24) by $\left(V_{\infty}^{m}\right)^{T}$, we obtain (21) again.

\subsection{Phase transition and epidemic threshold}

Many authors (see e.g. $[3,10,18,27]$ ) mention the existence of an epidemic threshold $\tau_{c}$. If the effective spreading rate $\tau=\frac{\beta}{\delta}>\tau_{c}$, the virus persists and a non-zero fraction of the nodes are infected, whereas for $\tau \leq \tau_{c}$, the epidemic dies out. The fact that the epidemic threshold occurs at $\tau=\tau_{c}=\frac{1}{\lambda_{1}}$ has been proved in several papers, see e.g. $[7,36]$. Here, we recall the fundamental lemma for the $N$-intertwined SIS model, proved in [36].

Lemma 2 There exists a value $\tau_{c}=\frac{1}{\lambda_{1}}>0$ and for $\tau<\tau_{c}$, there is only the trivial steady-state solution $V_{\infty}=0$. Beside the $V_{\infty}=0$ solution, there is a second, non-zero solution for all $\tau>\tau_{c}$. For $\tau=\tau_{c}+\varepsilon$, it holds that $V_{\infty}=\alpha x_{1}$, where $\varepsilon, \alpha>0$ are arbitrarily small constants and where $x_{1}$ is the eigenvector belonging to the largest eigenvalue $\lambda_{1}$ of the adjacency matrix $A$.

Lemma 2 has important practical consequences. Given a network with adjacency matrix $A$ and an imminent infection rate $\beta$, a nodal curing rate $\delta>\beta \lambda_{1}$ can be applied in nodes (in the form of anti-virus software or any other protection scheme) to maintain the network virus-free. A key-point is that the security of each host depends not only on the protection strategies it chooses to adopt but also on those chosen by other hosts in the network. In a heterogeneous setting, explained in Sect. 6, the resulting gametheoretic optimum has been studied in [26], while a different optimization technique in [16] minimizes the overall infection in the network by determining the individual curing rates of nodes. When the network can be modified, we possess a much larger number of ways to ban epidemics such as immunization strategies [8] and several ways to decrease the spectral radius $\lambda_{1}$ of the network: quarantining using the modular form of the network [24], degree-preserving rewiring [34,38,39] that changes the assortativity and modularity, and hence, the spectral radius. The optimal strategy to remove $m$ links from the network in order to minimize $\lambda_{1}$ is proved in [37] to be NP-hard. Consequently, several heuristics are proposed and evaluated in [37].

Lemma 2 shows that, for all graphs, $V_{\infty}=\alpha x_{1}+\xi y$, where $y$ is a vector orthogonal to $x_{1}, \alpha$ tends to zero as $\tau \downarrow \tau_{c}$, while $\xi$ tends faster to zero in that limit than $\alpha$. The following theorem is proved in [31]:

Theorem 2 For any graph with spectral radius $\lambda_{1}$ and corresponding eigenvector $x_{1}$ normalized such that $x_{1}^{T} x_{1}=\sum_{j=1}^{N}\left(x_{1}\right)_{j}^{2}=1$, the steady-state fraction of infected 
nodes $y_{\infty}$ obeys

$$
y_{\infty}(\tau)=\frac{1}{\lambda_{1} N} \frac{\sum_{j=1}^{N}\left(x_{1}\right)_{j}}{\sum_{j=1}^{N}\left(x_{1}\right)_{j}^{3}}\left(\tau_{c}^{-1}-\tau^{-1}\right)+O\left(\tau_{c}^{-1}-\tau^{-1}\right)^{2}
$$

when $\tau$ approaches the epidemic threshold $\tau_{c}$ from above.

Since the eigenvectors $x_{1}, x_{2}, \ldots, x_{N}$ belonging to the eigenvalues $\lambda_{1} \geq \lambda_{2} \geq$ $\cdots \geq \lambda_{N}$ of the adjacency matrix $A$ span the $N$-dimensional vector space, we can write the steady-state infection probability vector $V_{\infty}(\tau)$ as a linear combination of the eigenvectors of $A$,

$$
V_{\infty}(\tau)=\sum_{k=1}^{N} \gamma_{k}(\tau) x_{k}
$$

where the coefficient $\gamma_{k}(\tau)=x_{k}^{T} V_{\infty}(\tau)$ is the scalar product of $V_{\infty}(\tau)$ and the eigenvector $x_{k}$ and where the eigenvector $x_{k}$ obeys the normalization $x_{k}^{T} x_{k}=1$. Physically, (26) maps the dynamics $V_{\infty}(\tau)$ of the process onto the eigenstructure of the network, where $\gamma_{k}(\tau)$ determines the importance of the process in a certain eigendirection of the graph. The definition $y_{\infty}(\tau)=\frac{1}{N} u^{T} V_{\infty}(\tau)$ shows that

$$
y_{\infty}(\tau)=\frac{1}{N} \sum_{k=1}^{N} \gamma_{k}(\tau) u^{T} x_{k}
$$

Substitution of (26) into (16) yields

$$
y_{\infty}(\tau)=\frac{\tau}{N} \sum_{k=1}^{N} \lambda_{k} \gamma_{k}(\tau)\left(u^{T} x_{k}-\gamma_{k}(\tau)\right)
$$

For irregular graphs, generally, $\gamma_{m}(\tau)=x_{m}^{T} V_{\infty}(\tau) \neq 0$ for $m>1$ and all eigenvalues and eigenvectors in (28) play a role. Moreover, $\gamma_{m}(\tau)$ can be negative, as well as $\lambda_{m}$, while $\sum_{k=1}^{N} \lambda_{k}=0$ (see [32, p. 30]). The larger the spectral gap $\lambda_{1}-\lambda_{2}$ and the smaller $\left|\lambda_{N}\right|$, the more $y_{\infty}$ is determined by the dominant $k=1$ term in (28), and the more its viral behavior approaches that of a regular graph. Graphs with large spectral gap possess strong topological robustness [32], in the sense that it is difficult to tear that network apart.

Theorem 2 suggests, for all $1 \leq k \leq N$, the existence of the power series

$$
\gamma_{k}(\tau)=\sum_{j=1}^{\infty} c_{j}(k)\left(\tau_{c}^{-1}-\tau^{-1}\right)^{j}
$$


where $c_{1}(k)=0$ for $2 \leq k \leq N, c_{1}(1)=\left(\lambda_{1} \sum_{j=1}^{N}\left(x_{1}\right)_{j}^{3}\right)^{-1}$ and all other coefficients $c_{j}(k)$ can be determined in a recursive way as specified in the following lemma, which is proved in [33]:

\section{Lemma 3 Defining}

$$
X(m, l, k)=\sum_{q=1}^{N}\left(x_{m}\right)_{q}\left(x_{l}\right)_{q}\left(x_{k}\right)_{q}
$$

the coefficients $c_{j}(m)$ in (29) obey, for $m>1$ and $j>2$, the recursion

$$
\begin{aligned}
c_{j}(m)= & \frac{c_{j-1}(m)}{\lambda_{1}-\lambda_{m}}\left\{1-c_{1}(1)\left(\lambda_{1}+\lambda_{m}\right) X(m, m, 1)\right\} \\
& -\frac{c_{1}(1)}{\lambda_{1}-\lambda_{m}} \sum_{k=1 ; k \neq m}^{N}\left(\lambda_{1}+\lambda_{k}\right) c_{j-1}(k) X(m, k, 1) \\
& -\frac{1}{\lambda_{1}-\lambda_{m}} \sum_{n=2}^{j-2} \sum_{l=1}^{N} \sum_{k=1}^{N} c_{j-n}(l) c_{n}(k) \lambda_{k} X(m, l, k)
\end{aligned}
$$

while, for $j=2$ and $m>1$,

$$
c_{2}(m)=-\frac{1}{\lambda_{1}-\lambda_{m}} \frac{X(m, 1,1)}{\lambda_{1} X^{2}(1,1,1)}
$$

and $c_{1}(m)=0$. For $m=1$, there holds that $c_{1}(1)=\left(\lambda_{1} \sum_{j=1}^{N}\left(x_{1}\right)_{j}^{3}\right)^{-1}$ and for $j>1$, the coefficients $c_{j}$ (1) satisfy the recursion

$$
\begin{aligned}
c_{j}(1)= & -\frac{1}{\lambda_{1} X(1,1,1)} \sum_{k=2}^{N}\left(\lambda_{1}+\lambda_{k}\right) c_{j}(k) X(1,1, k) \\
& -\sum_{n=2}^{j-1} \sum_{l=1}^{N} \sum_{k=1}^{N} c_{j+1-n}(l) c_{n}(k) \lambda_{k} X(1, l, k)
\end{aligned}
$$

The radius of convergence of the Laurent series (11) and of the series (29) is, in general, unknown and still an open problem. From the definition (27), we obtain the series expansion of $y_{\infty}(\tau)$ around $\tau_{c}^{-1}-\tau^{-1}$ as

$$
y_{\infty}(\tau)=\sum_{j=1}^{\infty}\left\{\frac{1}{N} \sum_{k=1}^{N} c_{j}(k) u^{T} x_{k}\right\}\left(\tau_{c}^{-1}-\tau^{-1}\right)^{j}
$$




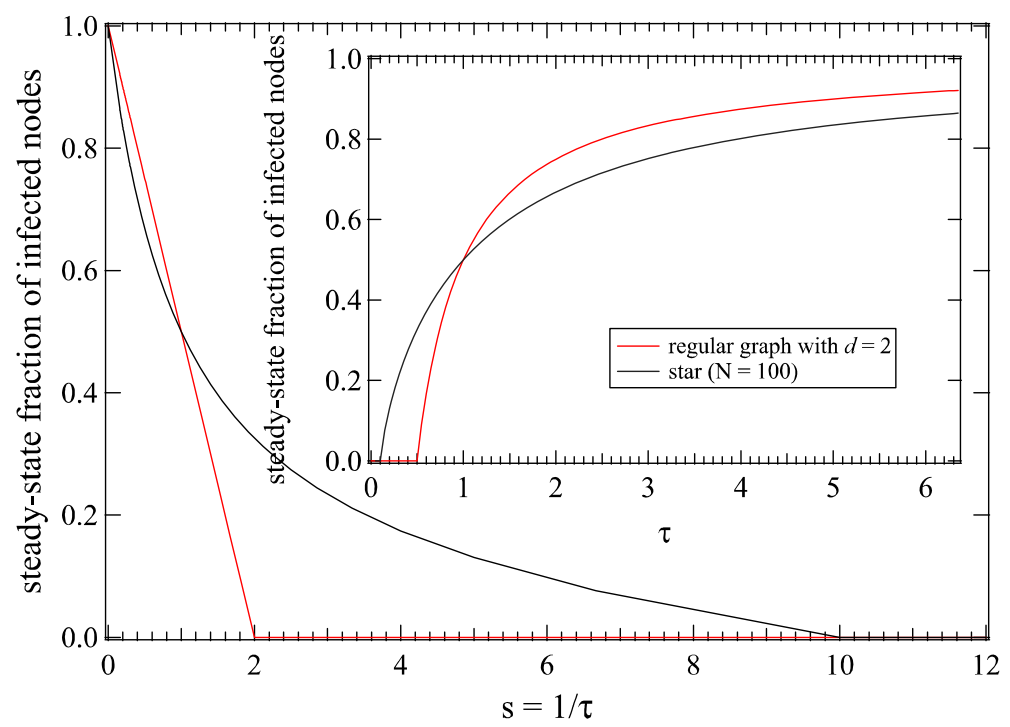

Fig. 3 The steady-state fraction $y_{\infty}$ of infected nodes versus $s=1 / \tau$ (and vs. $\tau$ in the inset) for a regular graph with degree $d=2$ and a star. Both graphs have $N=100$ nodes and almost the same average degree

valid for $\tau \geq \frac{1}{\lambda_{1}}$. Similarly, from the eigenvector expansion (26), all steady-state infection probabilities $v_{i \infty}(\tau)$ are expanded as

$$
v_{i \infty}(\tau)=\sum_{j=1}^{\infty}\left\{\sum_{k=1}^{N} c_{j}(k)\left(x_{k}\right)_{i}\right\}\left(\tau_{c}^{-1}-\tau^{-1}\right)^{j}
$$

Finally, Sahneh and Scoglio [11] have extended the $N$-intertwined model to three states: besides the susceptible and infected state, an alert state is introduced, that takes the behavior into account when a node realizes that it is surrounded by infected neighbors. The three-state extension is shown to exhibit two distinct epidemic thresholds. Between the two thresholds, the infection spreads at first, but eventually dies out due to increased alertness in the network.

\subsection{Transform $s=\frac{1}{\tau}$}

Figure 3 illustrates the typical behavior of $y_{\infty}(s)$ versus $s=\frac{1}{\tau}$ and $y_{\infty}(\tau)$ versus $\tau$ in the insert for a regular and irregular graph. Theoretically - though debatable -, one might argue that the effective curing rate $s=\frac{\delta}{\beta}=\frac{1}{\tau}$ is more natural than the effective infection rate $\tau=\frac{\beta}{\delta}$, because a Taylor expansion of $y_{\infty}(s)$ around $s=0$ exists, while the corresponding one (15) for $y_{\infty}(\tau)$ is a Laurent series in $\frac{1}{\tau}$ around $\tau \rightarrow \infty$. The Taylor expansion of $y_{\infty}(s)$ around $s_{c}=\lambda_{1}=\tau_{c}^{-1}$ follows directly from (30). 
4 Behavior of $y_{\infty}(s)$ around $s=\frac{E[D]}{2}$

In [25], we have shown, for any graph, that $y_{\infty} \leq \frac{1}{2}$ for $\tau \leq \frac{1}{E[D]}$. Moreover, simulations in [25] indicate that the maximum variance for the $N$-intertwined model is reached for $\tau \approx \frac{2}{E[D]}$.

Via extensive simulations, Youssef et al. [42] have observed that $y_{\infty} \simeq \frac{1}{2}$ around $s=\frac{E[D]}{2}$. In this section, several lemmas, proved in [33], explain and support these simulations.

Lemma 4 For any graph, it holds that

$$
y_{\infty}(s) \leq \frac{1}{2}+\frac{1}{2}\left(1-\frac{E[D]}{\lambda_{1}}\right) \text { for } s=\frac{E[D]}{2}
$$

and equality is only possible for the regular graph.

Lemma 5 For any graph, it holds that

$$
y_{\infty}(\tau) \leq \tau\left(\frac{E[D]}{4}+\frac{V_{\infty}^{T} Q V_{\infty}}{N}\right)
$$

and equality is only possible for the regular graph for which $V_{\infty}^{T} Q V_{\infty}=0$.

Lemma 5 shows for the regular graph that the "tangent" line through the origin $(\tau=0)$ lies above $y_{\infty}$; regular and only touches $y_{\infty}$; regular at the point $\tau=\frac{2}{E[D]}=\frac{2}{d}$. For any other graph, the slope is not larger than $\frac{E[D]}{4}+\frac{V_{\infty}^{T} Q V_{\infty}}{N}$ and, after transforming $s=\frac{1}{\tau}$ in (32), we find

$$
y_{\infty}(s) \leq \frac{1}{2}+\frac{2 V_{\infty}^{T} Q V_{\infty}}{N E[D]} \text { for } s=\frac{E[D]}{2}
$$

that complements (31). The correction $\frac{1}{2}\left(1-\frac{E[D]}{\lambda_{1}}\right)$ in (31) and the correction $\frac{2 V_{\infty}^{T} Q V_{\infty}}{N E[D]}$ in (33) are positive and small, but nevertheless show that $y_{\infty}(s)$ can be larger than $\frac{1}{2}$ at $s=\frac{E[D]}{2}$, as numerically found in [42].

Lemma 6 For any graph, it holds that

$$
\frac{y_{\infty}(\tau)}{\tau} \geq r(\tau)=\frac{1}{N} \sum_{j=1}^{N} v_{j \infty}\left(d_{j}-\lambda_{1} v_{j \infty}\right)
$$

where the lower bound obeys, for any $\tau$,

$$
r(\tau) \leq \frac{N_{2}}{4 N \lambda_{1}}
$$

and where $N_{k}=u^{T} A^{k} u$ denotes the number of walks of length $k$. 
We will now estimate the value $\xi$ for which $r(\xi)=\frac{N_{2}}{4 N \lambda_{1}}$ in irregular graphs where $\operatorname{Var}[D]>0$. Provided that there exists a value of $\tau=\xi$ for which $v_{j \infty}(\xi)=\frac{d_{j}}{2 \lambda_{1}}$ for each $1 \leq j \leq N$, that maximizes $r(\tau)$, we have that

$$
y_{\infty}(\xi)=\frac{1}{N} \sum_{j=1}^{N} \frac{d_{j}}{2 \lambda_{1}}=\frac{1}{2 \lambda_{1}} \frac{2 L}{N}=\frac{1}{2} \frac{E[D]}{\lambda_{1}}<\frac{1}{2}
$$

Lemma 6 then states that $y_{\infty}(\xi) \geq \frac{\xi N_{2}}{4 N \lambda_{1}}$ or

$$
\frac{1}{\lambda_{1}} \frac{L}{N} \geq \frac{\xi N_{2}}{4 N \lambda_{1}}
$$

from which

$$
\xi \leq \frac{4 L}{N_{2}}=2 \frac{E[D]}{(E[D])^{2}+\operatorname{Var}[D]}<\frac{2}{E[D]}
$$

In conclusion, there may exist a value $\xi$ such that $\tau_{c}<\xi<\frac{2}{E[D]}$ for which $y_{\infty}(\xi)=$ $\frac{1}{2} \frac{E[D]}{\lambda_{1}}<\frac{1}{2}$.

We end by deducing, approximately though, another type of lower bound. Concavity of $y_{\infty}(\tau)$ for $\tau \geq \tau_{c}=\frac{1}{\lambda_{1}}$ similarly leads to $y_{\infty}\left(q \tau_{c}+(1-q) m \tau_{c}\right) \geq$ $(1-q) y_{\infty}\left(m \tau_{c}\right)$. For sufficiently large $m$, the Laurent series (11) up to first order leads to

$$
y_{\infty}\left(m \tau_{c}\right) \geq 1-\frac{1}{m \tau_{c} N} \sum_{j=1}^{N} \frac{1}{d_{j}}
$$

because the second order term of $O\left(\frac{1}{\left(m \tau_{c}\right)^{2}}\right)$ is positive due to $\eta_{2}(i)>0$ in (13). Choosing $q \tau_{c}+(1-q) m \tau_{c}=\frac{2}{E[D]}$ provides us with

$$
\begin{aligned}
y_{\infty}\left(\frac{2}{E[D]}\right) & \geq \frac{\frac{2}{E[D]}-\tau_{c}}{(m-1) \tau_{c}}\left(1-\frac{1}{m \tau_{c} N} \sum_{j=1}^{N} \frac{1}{d_{j}}\right) \\
& >\left(\frac{2 \lambda_{1}}{E[D]}-1\right) \frac{1}{m}\left(1-\frac{\lambda_{1} E\left[\frac{1}{D}\right]}{m}\right)
\end{aligned}
$$

Ignoring the integer nature of $m$, the maximizer of the right-hand side occurs at $m=2 \lambda_{1} E\left[\frac{1}{D}\right]$, resulting in 


$$
y_{\infty}\left(\frac{2}{E[D]}\right) \gtrsim \frac{\frac{2 \lambda_{1}}{E[D]}-1}{4 \lambda_{1} E\left[\frac{1}{D}\right]}
$$

Notice that $\frac{\frac{2 \lambda_{1}}{E[D]}-1}{4 \lambda_{1} E\left[\frac{1}{D}\right]} \leq \frac{\frac{2 \lambda_{1}}{E[D]}-1}{4 \frac{\lambda_{1}}{E[D]}}<\frac{1}{2}$.

In summary, both last lower bound arguments illustrate, together with the upper bounds in Lemmas 4 and 5 that, for values of $\tau$ approaching $\frac{2}{E[D]}$, the steady-state fraction of infected nodes $y_{\infty}(\tau)$ is close to $\frac{1}{2}$ in any graph, in agreement with simulations [42].

\section{The viral conductance}

The viral conductance $\psi$ of a virus spreading process in a graph was first proposed in [20] as a new graph metric and then elaborated in more detail in [42]. The viral conductance $\psi$ is defined as

$$
\psi=\int_{0}^{\lambda_{1}} y_{\infty}(s) d s
$$

where $s=\frac{1}{\tau}$ and $\lambda_{1}$ is the spectral radius (i.e. the largest eigenvalue of the adjacency matrix $A$ of the graph) and equal to $s_{c}=\frac{1}{\tau_{c}}$. Below the epidemic threshold $\tau_{c}$, the network is virus-free in the steady-state. Hence, $v_{i \infty}(\tau)=0$ for $\tau<\tau_{c}$, and equivalently, $v_{i \infty}(s)=0$ for $s>\frac{1}{\tau_{c}}=\lambda_{1}$. Since the function $y_{\infty}(\tau)$ versus $\tau$ is not integrable over all $\tau$, Kooij et al. [20] have proposed to consider $y_{\infty}\left(\frac{1}{\tau}\right)$ versus $s=\frac{1}{\tau}$ (see Fig. 3).

In most published work so far, network $G_{1}$ was considered to be more robust against virus spread than network $G_{2}$ if the epidemic threshold $\tau_{c}\left(G_{1}\right)>\tau_{c}\left(G_{2}\right)$. For example, in Fig. 3, the regular graph with the same number $N$ of nodes and nearly the same number $L$ of links possesses a higher epidemic threshold than the star, and, thus, according to the above robustness criterion, the regular graph is more robust against virus propagation than the star. However, when the effective infection rate $\tau>1=2 \tau_{c}\left(G_{\text {regular }}\right)$, we observe from Fig. 3 that the percentage of infected nodes in the star is smaller than in the regular graph. The extent of the virus-free region is one aspect of the network's resilience against viruses, but once that barrier, the epidemic threshold, is crossed, the virus may conduct differently in networks with high and low epidemic threshold. This observation has led Kooij et al. [20] to propose the viral conductance as an additional metric.

The viral conductance $\psi$ is a graph metric that represents the overall conductance of the virus for all possible effective infection rates $\tau$ : when $\psi$ is high for the graph $G$, the virus can spread easily in $G$. Thus, instead of grading graphs only based on their epidemic threshold $\tau_{c}$ from virus vulnerable, where $\tau_{c}$ is small (high spectral radius $\lambda_{1}$ ) to virus robust (where $\tau_{c}$ is large), the viral conductance complements this classification with an average infection notion because 


$$
\overline{y_{\infty}}=\frac{1}{\lambda_{1}} \int_{0}^{\lambda_{1}} y_{\infty}(s) d s<1
$$

so that $\psi=\frac{\overline{y_{\infty}}}{\tau_{c}}<\frac{1}{\tau_{c}}=\lambda_{1}$. Graphs with small epidemic threshold may possess a small average fraction of infected nodes $\overline{y_{\infty}}$ so that the viral conductance can be equal to graphs with large epidemic threshold and large $\overline{y_{\infty}}$.

Using both expansions (15) and (30) into the definition (35) of $\psi$ yields, subject to the condition that the radius of convergence of both series is at least $\frac{\lambda_{1}}{2}$,

$$
\psi=\frac{\lambda_{1}}{2}-\frac{1}{N}\left\{\sum_{k=1}^{N} \frac{1}{d_{k}}-\frac{u^{T} x_{1}}{\lambda_{1} \sum_{j=1}^{N}\left(x_{1}\right)_{j}^{3}}\right\} \frac{\lambda_{1}^{2}}{8}+R
$$

where the remainder is

$$
R=\frac{1}{N} \sum_{m=j}^{\infty}\left\{\sum_{k=1}^{N} \eta_{m}(k)+c_{m}(k) u^{T} x_{k}\right\} \frac{\left(\lambda_{1}\right)^{m+1}}{2^{m+1}(m+1)}
$$

Subject to the above convergence condition, the expansion $R_{j}$ can be numerically computed up to any desired accuracy when the adjacency matrix $A$ is given, from which the eigenvectors $x_{1}, x_{2}, \ldots, x_{N}$ belonging to the eigenvalues $\lambda_{1} \geq \lambda_{2} \geq \cdots \geq \lambda_{N}$ can be computed.

Several bounds for the viral conductance are derived in [33] of which we only recall here a few. A simple upper bound, deduced from the convexity of $y_{\infty}(s)$ in $s \in\left[0, \lambda_{1}\right)$, is

$$
\psi \leq \frac{\lambda_{1}}{2}
$$

More accurate lower and upper bounds are

$$
\psi \geq \frac{\lambda_{1}}{2}\left\{Z+\zeta(1-Z)+\frac{\min _{s \in\left[0, \lambda_{1}\right]} y_{\infty}^{\prime \prime}(s)}{3}\left\{\lambda_{1}^{2}-3 \lambda_{1} \zeta+3 \zeta^{2}\right\}\right\}
$$

and

$$
\psi \leq \frac{\lambda_{1}}{2}\left\{Z+\zeta(1-Z)+\frac{\max _{s \in\left[0, \lambda_{1}\right]} y_{\infty}^{\prime \prime}(s)}{3}\left\{\lambda_{1}^{2}-3 \lambda_{1} \zeta+3 \zeta^{2}\right\}\right\}
$$

where $Z=\frac{1}{N} \frac{\sum_{j=1}^{N}\left(x_{1}\right)_{j}}{\sum_{j=1}^{N}\left(x_{1}\right)_{j}^{3}}<1$ and $\zeta=\frac{1-Z}{\lambda_{1} E\left[\frac{1}{D}\right]-Z} \leq 1$. Other types of bounds are

$$
\frac{d_{\min }}{2} \leq \psi<\ln \left(1+\frac{\lambda_{1}}{d_{\min }-1}\right)\{E[D]-1\}
$$


The largest possible ratio $\frac{\lambda_{1}}{d_{\min }}=O(\sqrt{N})$ occurs in the star, which illustrates that the viral conductance is bounded by $\frac{1}{2} E[D] \log N$. Likely, the star possesses the largest viral conductance among all graphs with $N$ nodes and $L$ links. It is further conjectured in [42] that the regular graph attains the lowest viral conductance all graphs with $N$ nodes and $L$ links. This conjecture has only partially been proved so far in [33] to be true for the class of complete bipartite graphs, but not yet for all graphs.

\section{Heterogeneous $N$-intertwined model}

The homogenous $N$-intertwined model, where the infection and curing rate is the same for each link and node in the network, has been extended to a heterogeneous setting in [35], where an infected node $i$ can infect its neighbors with an infection rate $\beta_{i}$, but it is cured with curing rate $\delta_{i}$.

Heterogeneity rather than homogeneity abounds in real networks. For example, in data communications networks, the transmission capacity, age, performance, installed software, security level and other properties of networked computers are generally different. Social and biological networks are very diverse: a population often consists of a mix of weak and strong, or old and young species or of completely different types of species. The network topologies for transport by airplane, car, train, ship are different. Many more examples can be added illustrating that homogeneous networks are the exception rather than the rule. This diversity in the "nodes" and "links" of real networks will thus likely affect the spreading pattern of viruses. In previous Sections, only a homogeneous virus spread was investigated, where all infection rates $\beta_{i}=\beta$ and all curing rates $\delta_{i}=\delta$ were the same for each node. We believe that the extension to a full heterogeneous setting is, perhaps, the best SIS model that we can achieve.

The governing differential equation (1) is straightforwardly generalized to

$$
\frac{d v_{i}(t)}{d t}=\sum_{j=1}^{N} \beta_{j} a_{i j} v_{j}(t)-v_{i}(t)\left(\sum_{j=1}^{N} \beta_{j} a_{i j} v_{j}(t)+\delta_{i}\right)
$$

while the corresponding matrix equation is

$$
\frac{d V(t)}{d t}=\operatorname{Adiag}\left(\beta_{j}\right) V(t)-\operatorname{diag}\left(v_{i}(t)\right)\left(A \operatorname{diag}\left(\beta_{j}\right) V(t)+C\right)
$$

where $\operatorname{diag}\left(v_{i}(t)\right)$ is the diagonal matrix with elements $v_{1}(t), v_{2}(t), \ldots, v_{N}(t)$ and the curing rate vector is $C=\left(\delta_{1}, \delta_{2}, \ldots, \delta_{N}\right)$. We note that $A \operatorname{diag}\left(\beta_{i}\right)$ is, in general and opposed to the homogeneous setting, not symmetric anymore, unless $A$ and $\operatorname{diag}\left(\beta_{i}\right)$ commute, in which case the eigenvalue $\lambda_{i}\left(A \operatorname{diag}\left(\beta_{i}\right)\right)=\lambda_{i}(A) \beta_{i}$ and both $\beta_{i}$ and $\lambda_{i}(A)$ have a same eigenvector $x_{i}$. 


\subsection{The steady-state}

The metastable steady-state follows from (38) as

$$
A \operatorname{diag}\left(\beta_{i}\right) V_{\infty}-\operatorname{diag}\left(v_{i \infty}\right)\left(A \operatorname{diag}\left(\beta_{i}\right) V_{\infty}+C\right)=0
$$

where $V_{\infty}=\lim _{t \rightarrow \infty} V(t)$. We define the vector

$$
w=A \operatorname{diag}\left(\beta_{i}\right) V_{\infty}+C
$$

and write the stead-state equation as

$$
w-C=\operatorname{diag}\left(v_{i \infty}\right) w
$$

or

$$
\left(I-\operatorname{diag}\left(v_{i \infty}\right)\right) w=C
$$

Ignoring extreme virus spread conditions (the absence of curing $\left(\delta_{i}=0\right)$ and an infinitely strong infection rate $\left.\beta_{i} \rightarrow \infty\right)$, then the infection probabilities $v_{i \infty}$ cannot be one such that the matrix $\left(I-\operatorname{diag}\left(v_{i \infty}\right)\right)=\operatorname{diag}\left(1-v_{i \infty}\right)$ is invertible. Hence,

$$
w=\operatorname{diag}\left(\frac{1}{1-v_{i \infty}}\right) C
$$

Invoking the definition (39) of $w$, we obtain

$$
\begin{aligned}
\operatorname{Adiag}\left(\beta_{i}\right) V_{\infty} & =\operatorname{diag}\left(\frac{v_{i \infty}}{1-v_{i \infty}}\right) C \\
& =\operatorname{diag}\left(\frac{\delta_{i}}{1-v_{i \infty}}\right) V_{\infty}
\end{aligned}
$$

that generalizes (24). The $i$-th row of (40) yields the nodal steady state equation,

$$
\sum_{j=1}^{N} a_{i j} \beta_{j} v_{j \infty}=\frac{v_{i \infty} \delta_{i}}{1-v_{i \infty}}
$$

Let $\widetilde{V}_{\infty}=\operatorname{diag}\left(\beta_{i}\right) V_{\infty}$ and the effective spreading rate for node $i, \tau_{i}=\frac{\beta_{i}}{\delta_{i}}$, then we arrive at

$$
\mathcal{Q}\left(\frac{1}{\tau_{i}\left(1-v_{i \infty}\right)}\right) \widetilde{V}_{\infty}=0
$$


where the symmetric matrix

$$
\begin{aligned}
\mathcal{Q}\left(q_{i}\right) & =\operatorname{diag}\left(q_{i}\right)-A \\
& =\operatorname{diag}\left(q_{i}-d_{i}\right)+Q
\end{aligned}
$$

can be interpreted as a generalized Laplacian ${ }^{1}$, because $\mathcal{Q}\left(d_{i}\right)=Q=\Delta-A$, where $\Delta=\operatorname{diag}\left(d_{i}\right)$. The observation that the non-linear set of steady-state equations can be written in terms of the generalized Laplacian $\mathcal{Q}\left(q_{i}\right)$ is fortunate, because the powerful theory of the "normal" Laplacian $Q$ applies. Many properties of the generalized Laplacian $\mathcal{Q}\left(q_{i}\right)$ are given in [35] that enabled to prove three important theorems. The first theorem is

Theorem 3 The critical threshold is determined by vectors $\tau_{c}=\left(\tau_{1 c}, \tau_{2 c}, \ldots, \tau_{N c}\right)$ that obey $\lambda_{\max }(R)=1$, where $\lambda_{\max }(R)$ is the largest eigenvalue of the symmetric matrix

$$
R=\operatorname{diag}\left(\sqrt{\tau_{i}}\right) \operatorname{Adiag}\left(\sqrt{\tau_{i}}\right)
$$

whose corresponding eigenvector has positive components if the graph $G$ is connected.

Several bounds for $\lambda_{\max }(R)$ are derived and $\lambda_{\max }(R)$ for the complete graph $K_{N}$ is solved exactly. The generalization of Theorem 1 is

Theorem 4 The non-zero steady-state infection probability of any node $i$ in the $N$-intertwined model can be expressed as a continued fraction

$$
v_{i \infty}=1-\frac{1}{1+\frac{\gamma_{i}}{\delta_{i}}-\delta_{i}^{-1} \sum_{j=1}^{N} \frac{\beta_{j} a_{i j}}{1+\frac{\gamma_{j}}{\delta_{j}}-\delta_{j}^{-1} \sum_{k=1}^{N} \frac{\beta_{k} a_{j k}}{1+\frac{\gamma_{k}}{\delta_{k}}-\delta_{k}^{-1} \sum_{q=1}^{N} \frac{a_{q k} \beta_{q}}{\ddots}}}}
$$

where the total infection rate of node $i$, incurred by all neighbors towards node $i$, is

$$
\gamma_{i}=\sum_{j=1}^{N} a_{i j} \beta_{j}=\sum_{j \in \text { neighbor }(i)} \beta_{j}
$$

Consequently, the exact steady-state infection probability of any node $i$ is bounded by

$$
0 \leq v_{i \infty} \leq 1-\frac{1}{1+\frac{\gamma_{i}}{\delta_{i}}}
$$

Perhaps the most important theorem proved in [35] is

\footnotetext{
${ }^{1}$ All eigenvalues of the Laplacian $Q=\Delta-A$ in a connected graph are positive, except for the smallest one that is zero. Hence, $Q$ is positive semi-definite. Much more properties of the Laplacian $Q$ are found e.g. in $[5,9,32]$.
} 
Theorem 5 Given that all curing rates $\delta_{j}$ for $1 \leq j \neq i \leq N$ are constant and independent from the infection rates $\beta_{j}$, the non-zero steady-state infection probability $v_{i \infty}\left(\delta_{1}, \ldots, \delta_{i}, \ldots, \delta_{N}\right)>0$ is strict convex in $\delta_{i}$, while all other non-zero steady-state infection probabilities $v_{j \infty}\left(\delta_{1}, \ldots, \delta_{i}, \ldots, \delta_{N}\right)>0$ are concave in $\delta_{i}$.

A direct consequence of Theorem 5 to the homogeneous setting is that $y_{\infty}(s)$ is convex for $s \in\left[0, \lambda_{1}\right)$ (or $y_{\infty}(\tau)$ is concave for $\tau>\tau_{c}$ ).

\section{Conclusion}

The $N$-intertwined SIS network model has been introduced and many derived results in the steady-state have been reviewed. While extensions of the model are certainly expected in the future, we believe that the steady-state theory of the homogeneous $N$-intertwined SIS network model is almost entirely established. The time-dependent theory, on the other hand, needs much more efforts towards maturity. Although the $\mathrm{N}$-intertwined model is not exact, the only — a mean-field — approximation has enabled analytic computations as presented here that are, to the best of our knowledge, not possible with any other SIS model that is more accurate than the $N$-intertwined model. An open problem is to determine of the overall accuracy of the $N$-intertwined model (with respect to the exact SIS Markov process) for any value of $\tau$ in a broad class of interesting networks. So far, numerical simulations [21] point to a promisingly good accuracy that improves with $N$.

A newly envisioned direction is the coupling of the virus spread process with the underlying topology. In other words, the presented model has assumed that the adjacency matrix $A$ is fixed and is not changed by the virus spread process. Hence, nodes can only protect themselves against the virus by increasing their curing rate $\delta$. While taking medicine or vaccination is one measure in the fight against the virus, a more natural one is to avoid contact with infected people. The latter assumes that the adjacency matrix $A$ is changed by the process and the knowledge that a node's neighbor(s) is (are) infected. The precise description of the coupling between virus spread process and topology as well as the solution of the far more complex set of differential equations stand on the agenda of future research.

Acknowledgements We are very grateful to Caterina Scoglio, Mina Youssef, Faryad Darabi Sahneh, Cong Li and Rob Kooij for many comments on an early version of the manuscript. This research was supported by Next Generation Infrastructures (Bsik) and the EU FP7 project ResumeNet (project No. 224619).

Open Access This article is distributed under the terms of the Creative Commons Attribution Noncommercial License which permits any noncommercial use, distribution, and reproduction in any medium, provided the original author(s) and source are credited.

\section{References}

1. Anderson RM, May RM (1991) Infectious diseases of humans: dynamics and control. Oxford University Press, Oxford

2. Asavathiratham C (2000) The influence model: a tractable representation for the dynamics of networked markov chains. Ph.D thesis, Massachusetts Institute of Technology, Cambridge 
3. Bailey NTJ (1975) The mathematical theory of infectious diseases and its applications, 2nd edn. Charlin Griffin \& Company, London

4. Barrat A, Bartelemy M, Vespignani A (2008) Dynamical processes on complex networks. Cambridge University Press, Cambridge

5. Biggs N (1996) Algebraic graph theory, 2nd edn. Cambridge University Press, Cambridge

6. Castellano C, Pastor-Satorras R (2010) Thresholds for epidemic spreading in networks. Phys Rev Lett 105:218701

7. Chakrabarti D, Wang Y, Wang C, Leskovec J, Faloutsos C (2008) Epidemic thresholds in real networks. ACM Trans Inf Syst Secur (TISSEC) 10(4):1-26

8. Chen Y, Paul G, Havlin S, Liljeros F, Stanley HE (2008) Finding a better immunization strategy. Phys Rev Lett 101:058701

9. Cvetković DM, Doob M, Sachs H (1995) Spectra of fraphs, theory and applications, 3rd edn. Johann Ambrosius Barth Verlag, Heidelberg

10. Daley DJ, Gani J (1999) Epidemic modelling: an introduction. Cambridge University Press, Cambridge

11. Darabi Sahneh F, Scoglio C (2011) Epidemic spread in human networks. In: 50th IEEE conference on decision and control, Orlando, December 2011. arXiv:1107.2464v1

12. Durrett R (2010) Some features of the spread of epidemics and information on a random graph. Proc Natl Acad Sci USA (PNAS) 107(10):4491-4498

13. Ganesh A, Massoulié L, Towsley D (2005) The effect of network topology on the spread of epidemics. In: IEEE INFOCOM05, San Francisco

14. Garetto M, Gong W, Towsley D (2003) Modeling malware spreading dynamics. In: IEEE INFOCOM'03, San Francisco, April 2003

15. Gómez S, Arenas A, Borge-Holthoefer J, Meloni S, Moreno Y (2010) Discrete-time Markov chain approach to contact-based disease spreading in complex networks. Europhys Lett (EPL) 89:38009

16. Gourdin E, Omic J, Van Mieghem P (2011) Optimization of network protection against virus spread. In: 8th international workshop on design of reliable communication networks (DRCN 2011), Krakow, 10-12 October 2011

17. Hill AL, Rand DG, Nowak MA, Christakis NA (2010) Emotions as infectious diseases in a large social network: the SISa model. Proc Royal Soc B 277:3827-3835

18. Kephart JO, White SR (1991) Direct-graph epidemiological models of computer viruses. In: Proceedings of the 1991 IEEE computer society symposium on research in security and privacy, pp 343-359, May 1991

19. Kermack WO, McKendrick AG (1927) A contribution to the mathematical theory of epidemics. Proc Royal Soc A 115:700-721

20. Kooij RE, Schumm P, Scoglio C, Youssef M (2009) A new metric for robustness with respect to virus spread. In: Networking 2009, LNCS 5550, pp 562-572

21. Omic J (2010) Epidemics in networks: modeling, optimization and security games. Ph.D thesis, September 2010. http://repository.tudelft.nl/. Accessed Sept 2010

22. Omic J, Kooij RE, Van Mieghem P (2007) Virus spread in complete bi-partite graphs. In: Bionetics 2007, Budapest, 10-13 December 2007

23. Omic J, Kooij RE, Van Mieghem P (2009) Heterogeneous protection in regular and complete bi-partite networks. In: IFIP networking 2009, Aachen, 11-15 May 2009

24. Omic J, Martin Hernandez J, Van Mieghem P (2010) Network protection against worms and cascading failures using modularity partitioning. In: 22nd international teletraffic congress (ITC 22), Amsterdam, 7-9 September 2010

25. Omic J, Van Mieghem P (2009) Epidemic spreading in networks_-variance of the number of infected nodes. Delft University of Technology, report20090707. http://www.nas.ewi.tudelft.nl/people/Piet/ TUDelftReports

26. Omic J, Van Mieghem P, Orda A (2009) Game theory and computer viruses. In: IEEE Infocom09

27. Pastor-Satorras R, Vespignani A (2001) Epidemic spreading in scale-free networks. Phys Rev Lett 86(14):3200-3203

28. Restrepo JG, Ott E, Hunt Brian R (2005) Onset of synchronization in large networks of coupled oscillators. Phys Rev E 71(036151):1-12

29. Strogatz SH (2000) From Kuramoto to Crawford: exploring the onset of synchronization in populations of coupled oscillators. Physica D 143:1-20

30. Van Mieghem P (2006) Performance analysis of communications systems and networks. Cambridge University Press, Cambridge 
31. Van Mieghem P (2011) Epidemic phase transition of the SIS-type in networks (submitted)

32. Van Mieghem P (2011) Graph spectra for complex networks. Cambridge University Press, Cambridge

33. Van Mieghem P (2011) Viral conductance of a network (submitted)

34. Van Mieghem P, Ge G, Schumm P, Trajanovski S, Wang H (2010) Spectral graph analysis of modularity and assortativity. Phys Rev E 82:056113

35. Van Mieghem P, Omic J (2008) In-homogeneous virus spread in networks. Delft University of Technology, Report2008081. http://www.nas.ewi.tudelft.nl/people/Piet/TUDelftReports

36. Van Mieghem P, Omic J, Kooij RE (2009) Virus spread in networks. IEEE/ACM Trans Netw 17(1): $1-14$

37. Van Mieghem P, Stevanović D, Kuipers FA, Li C, van de Bovenkamp R, Liu D, Wang H (2011) Decreasing the spectral radius of a graph by link removals. Phys Rev E 84(1):016101

38. Van Mieghem P, Wang H, Ge X, Tang S, Kuipers FA (2010) Influence of assortativity and degree-preserving rewiring on the spectra of networks. E Phys J B 76(4):643-652

39. Wang H, Winterbach W, Van Mieghem P (2011) Assortativity of complementary graphs. Eur Phys J B (to appear)

40. Wang Y, Chakrabarti D, Wang C, Faloutsos C (2003) Epidemic spreading in real networks: an eigenvalue viewpoint. In: 22nd international symposium on reliable distributed systems (SRDS'03), IEEE computer, pp 25-34, October 2003

41. Wang Y, Wang C (2003) Modeling the effects of timing parameters on virus propagation. In: ACM workshop on rapid malcode (WORM'03), Washington DC, pp 61-66, 27 Oct 2003

42. Youssef M, Kooij RE, Scoglio C (2011) Viral conductance: quantifying the robustness of networks with respect to spread of epidemics. J Comput Science. doi:10.1016/j.jocs.2011.03.001

43. Youssef M, Scoglio C (2011) An individual-based approach to SIR epidemics in contact networks. J Theor Biol 283:136-144 